Solid State Communications, Vol. 47, No. 5, pp. 383-386, 1983.

Printed in Great Britain.

$0038-1098 / 83 \$ 3.00+.00$

Pergamon Press Ltd.

\title{
QUANTUM TRANSPORT OF ELECTRONS CONFINED IN A THIN GaAs LAYER BY AN IMPURITY SPACE CHARGE POTENTIAL IN HIGH MAGNETIC FIELDS
}

\author{
J.C. Maan, Th. Englert and Ch. Uihlein \\ Max Planck Institut für Festkörperforschung. Hochfeld Magnetlabor 166X. F38042 Grenoble Cedex, France \\ and \\ H. Künzel, A. Fischer and K. Ploog
}

Max Planck Institut für Festkörperforschung, 7000 Stuttgart 80. Federal Republic of Germany

(Received 14 April 1983 by M. Balkanski)

The resistance of a thin, $n$-doped, GaAs layer sandwiched between $p$-doped layers shows clear quantum oscillations as a function of the magnetic field which depend on the orientation of the magnetic field with respect to the layer plane. The data for parallel and perpendicular magnetic fields are analyzed in terms of two dimensional conductivity using a simple model and the subband structure is deduced consistently from both experiments.

THE PROPERTIES of two-dimensional electronic systems in semiconductors have been extensively studied in the past decade [1]. Space quantization has been realized most frequently in interfaces of heterostructures, in thin layers of semiconductors and in space charge layers. Recently the first experimental evidence of the existence of a two-dimensional electron gas (2 DEG) confined by an impurity space charge potential has been reported [2]. This idea was originally proposed by Esaki and Tsu [3] and was theoretically developed in great detail by Döhler [4]. Several interesting theoretical predictions $[4]$ have been confirmed experimentally, such as the existence of 2.D subbands [2], the tunability of the effective band gap [2], of the optical absorption coefficient [5] and of the carrier density [6].

In this paper we report conductivity measurements of a thin $n$-type GaAs layer sandwiched between $p$-type layers in GaAs in strong magnetic fields $(B)$ for orientations of $B$ parallel and perpendicular to the plane of the $n$-layer. From the observed oscillations of the sumple resistance in both magnetic field orientations and Hall effect measurements, the separation between subbands is derived using a simple model for the subband structure. The subband separation is found to decrease with increasing carrier density, which was varied through the application of a voltage $U_{n p}$ between the $n$ - and the $p$ layers. The experiments confirm the 2-D nature of the electronic transport in the $n$-layer. Preliminary results of magneto-conductivity measurements on a doping superlattice with 10 periods of $n$ - and $p$-layers have been reported elsewhere [7]. In that case only the perpendicular magnetic field data were analyzed in detail. In order to reconcile the Shubnikov de Haas $(\mathrm{SdH})$ result and the density obtained from the Hall effect a correction factor had to be assumed. This observed discrepancy may be explained by contact problems related to the multilayer nature of that sample.

In a mp structure the electrons from the donors transfer to the acceptors in the $p$-layers. The resulting space charge leads to a potential well confining the elec. trons in the centre of the $n$-layer. Through selective contacts to the $p$-and the $n$-layers, the potential difference between these layers can be varied externally. This way the carrier density in the $n$-layer can be modulated provided that the $n$-layer thickness is of the order of twice the depletion layer thickness [6]. Since the electron and the holes are spatially separated, the recombination current at low temperatures is negligibly small for reversed bias and for forward bias as long as the applied voltage $U_{n p}$ times the elementary charge is small com. pared to the bandgap. Therefore the change in the carrier density can be considered quasi static. Without external voltage the depletion width $d_{\text {depl }}$ in the $n$-layer at the $p n$ junction is approximately given by:

$d_{\mathrm{depl}}=\left[2 \epsilon E_{G} N_{A} / e^{2} N_{D}\left(N_{D}+N_{A}\right)\right]^{1 / 2}$,

where $E_{G}$ is the bandgap $\epsilon$ the dielectric constant, $N_{D}$ the donor and $N_{A}$ the acceptor concentration. If $d_{\text {depl }}$ is exactly half the doping layer thickness the electrons are confined in a parabolic potential well and equally spaced 2-D subbands are formed with a separation of $\left(e^{2} N_{D} / \epsilon m^{*}\right)^{1 / 2}$. With a positive (negative) voltage $U_{n p}$ carriers are injected (extracted) and therefore impurities are neutralized (ionized) which decreases (increases) the space charge potential leading to a decrease (increase) of the subband separation. 


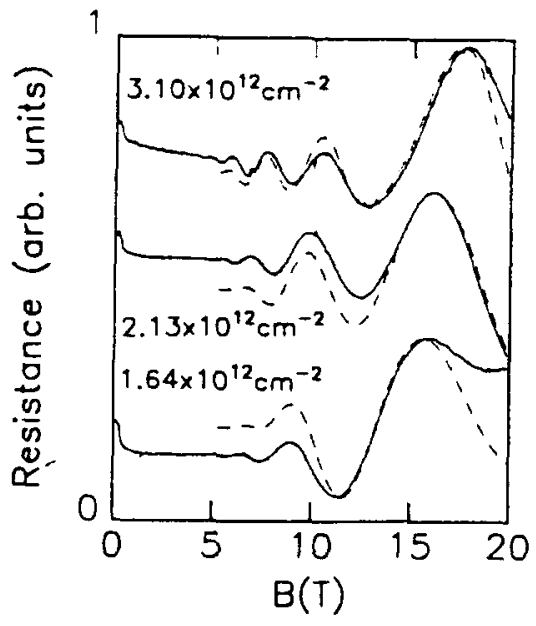

Fig. 1. Measured sample resistance as a function of the magnetic field for three different carrier densities (drawn lines) with the magnetic field perpendicular to the layer. The dashed curves represent the theoretical fit as described in the text.

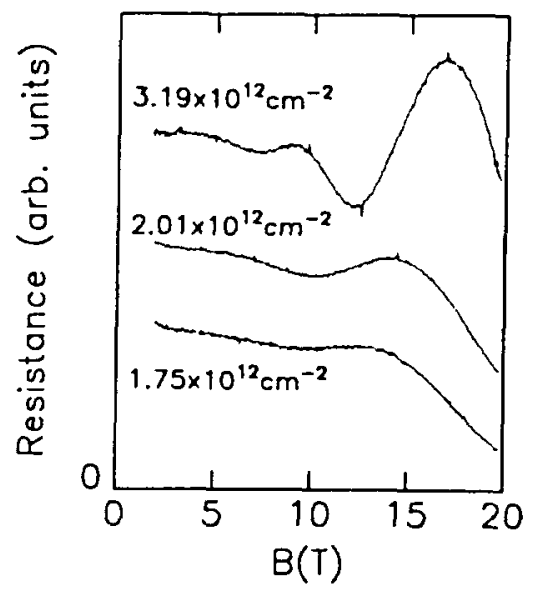

Fig. 2. Measured sample resistance as a function of the magnetic field parallel to the layer plane for three different densities.

The sample was grown by the technique of Molecular Beam Epitaxy [8] and had the usual Hall bar shape for the transport measurements. Selective $n$ and $p$ contacts were diffused in the layers and were arranged in such a way that the Hall voltage and the resistance of the $n$-channel could be measured as a function of $U_{n p}$. The parameters of the present sample were: thickness of the $p$-type cladding layers $500 \mathrm{~nm}$ and of the central $n$-layer $90 \mathrm{~nm}$ with $N_{D}=N_{A}=7 \times 10^{17} \mathrm{~cm}^{-3}$ and an electron mobility of $\left(2200 \mathrm{~cm}^{2} \mathrm{Vsec}^{-1}\right)$ at $4 \mathrm{~K}$.

The results of the magnetoresistance measurements with the magnetic field perpendicular to the layer plane

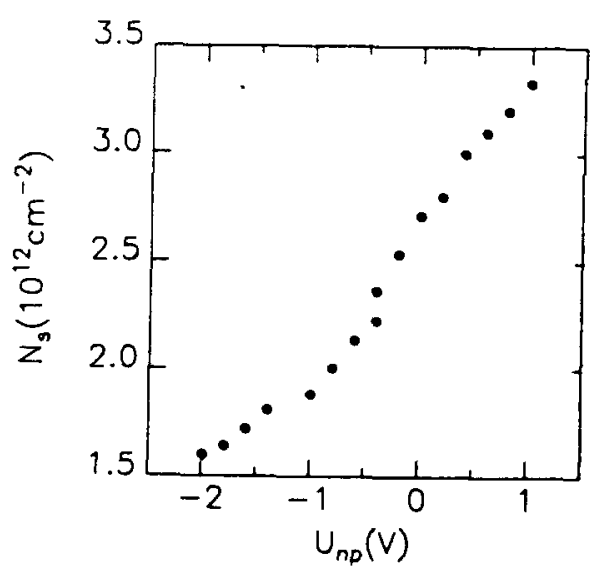

Fig. 3. Measured change in the carrier density as a function of the voltage $U_{n p}$ between the $n$ and the $p$-layers.

are shown in Fig. 1 for three different carrier densities. In Fig. 2 the result for $B$ parallel to the layer plane are plotted. The current was always perpendicular to the magnetic field. In both cases well pronounced quant um oscillations are observed but for $B_{\|}$the amplitude is weaker especially at lower fields and the strong maxi. mum at high $B$ values is shifted to lower magnetic fields with respect to the $B_{1}$ data. These results clearly show an anisotropy which is incompatible with the threedimensional GaAs conduction band structure and which demonstrates directly the two-dimensional nature of the conductance in the $n$-channel. Figure 3 shows the chang, in the carrier density as determined from the Hall effect as a function of voltage $U_{n p}$.

We next analyze the data for $B_{\|}$and $B_{1}$ and derive the separation between subbands from both experiments. For this purpose we approximate the space charge potential profile by a parabolic potential well (harmonic oscillator). This approximation is certainly valid for low carrier densities as explained before. Moreover, theoretical calculations, which include the influenc, of the free electrons on the space charge potential self. consistently, have shown that the subbands remain in good approximation equidistant even at higher densities $[9,10]$. The advantage of our approximation is that analytical solutions can be obtained for the subband structure in a parallel magnetic field. The Hamiltonian for the electrons in the $n$-layer in a magnetic field is then given by:

$H=(\mathrm{p}+e \mathrm{~A})^{2}+F z^{2} / 2$,

where $\mathbf{A}=\left(0, x B_{z},-z B_{x}\right)$ the vector potential and $F$ parametrizes the harmonic potential (the doping gradient is taken in the $z$-direction $)$. For $B_{x}=0\left(B_{1}\right.$ to the layer) equation (1) leads to a set of Landau levels for each subband having a degeneracy of $2 e B / h$ each; 


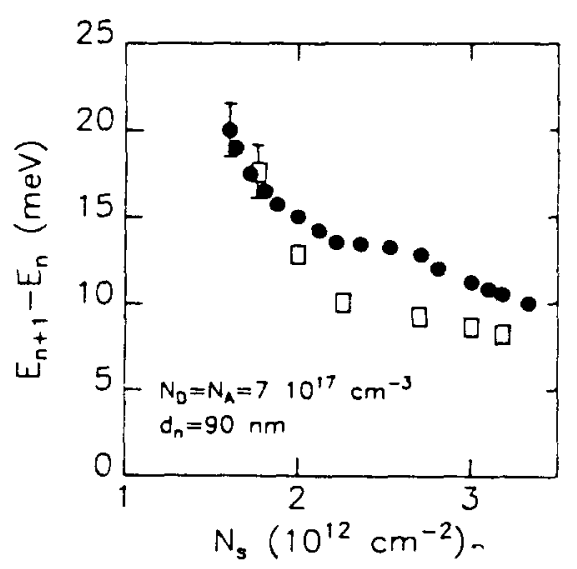

Fig. 4. Subband separation as derived from the experiments with $B$ perpendicular (circles) and $B$ parallel to the layer plane (squares).

$E_{N, n}=(N+1 / 2) h \omega_{c}+(n+1 / 2) h \omega_{F}$,

where $N$ is the Landau level index, $n$ the subband quan. tum number, $\omega_{c}=c B_{\text {tot }} / m^{*}$ and $\omega_{F}=\left(F / m^{*}\right)^{1 / 2}$. For $B_{z}=0(B$ parallel to the layer plane) equation I leads to a set of subbands $E_{n}\left(k_{x}, k_{y}\right)$ :

$$
\begin{aligned}
& E_{n}\left(k_{x}, k_{y}\right)=(n+1 / 2) h\left(\omega_{c}^{2}+\omega_{F}^{2}\right)^{1 / 2} \\
& +h^{2} / 2 m^{*}\left|k_{x}^{2}+k_{y}^{2}\left\{\omega_{F}^{2} /\left(\omega_{c}^{2}+\omega_{F}^{2}\right)\right\}^{1 / 2}\right| .
\end{aligned}
$$

The subband energy increases with $B_{\|}$and the density of states $g(f)$ in cach subband is given by:

$g(\xi) \mathrm{d} l:=m^{*} / \pi h^{2}\left|\omega_{F}^{2} /\left(\omega_{c}^{2}+\omega_{k}^{2}\right)\right|^{t / 2} \mathrm{~d} E ;$

and increases also with the magnetic lield. Therefore the density of states, and consequently the origin of the quantum oscillations is entirely different for both orientations. For $B_{\perp}$ the oscillations are essentially due to variations in the intra Landau level scattering rate as the Landau levels cross the Fermi energy with increasing magnetic field and retlect the density of states (normal Sdn effect). For $B_{\|}$the oscillations in a quasi 2-D system are generally explained in terms of a varying intersubband scattering rate since the subbands shift to higher energy and since their density of states increases with $B_{\|}[11,12]$. Consequently the carriers can be accomodated in less subbands and the intersubband scattering is reduced each time a subband moves through the Fermi energy.

For the $B_{1}$ measurements we simulate the experimental results with a model for the two-dimensional conductivity based upon the theory of Ando and Uemura [13] which was successfully employed to explain experimental data from the GaAs/Gal ${ }_{1-x} A l_{x}$ As heterojunction [14]. This model is extended in a simple manner to allow for the occupation of more subbands. The conductivity tensor element $\sigma_{x x}$ can be written as: $\sigma_{x x} \propto \int\left(-\frac{\partial f}{\partial E}\right) \sum_{n} \sum_{N}(N+1 / 2) \exp \left[\left(\frac{E-E_{n}-E_{N}}{\Gamma}\right)\right]^{2} \mathrm{~d} E$.

where $\Gamma$ is a level broadening parameter (a Gaussian broadening is assumed) and $f$ the Fermi function. The tensor relation has been used to calculate $\rho_{x x}$ and $\sigma_{x x}$ and $\rho_{x y}$ is taken to be $N_{s} e / B$ where $N_{s}$ is the 2-D carrier density as determined from the Hall effect. The subband separation $E_{n+1}-E_{n}=h \omega_{F}$ is treated as a fit parameter. Representative results for the fit are shown as the dotted lines in Fig. 1. Qualitatively the model reproduces the experimental results remarkably well as witnessed by the good agreement of the position and the amplitude of the observed and calculated extrema. $E_{n+1}-E_{n}$ as a function of the carrier density obtained in this way is shown in Fig. 4 (circles) and is found to decrease with increasing carrier density as expected. It should be noted that the uscillatory pattern does not change drastically as the carrier density increases by a factor two. We explain this by the fact that the observed SdH oscillations result from a superposition of different subbands and that their period in this case is not related to the total 2.D carrier density in a simple manner. As a consquence of the simulaneous decreases in the subband separation with increasing carrier density the oscillations move only slightly to higher magnetic fields.

For $B_{1 f}$ it is not a priori clear which feature in the spectra corresponds to the situation where al subband moves through the Fermi energy. We have found that we can obtain good agreement between the subband structure obtained for $B_{\perp}$ and $B_{\|}$if we assume that a maxi. mum in the resistance is associated with the case that the Fermi energy is at the edge of a subband. Qualitatively one can argue that the resistance will decrease when a subband is emptied at increasing magnetic field, since the intersubband scattering will be reduced. Furthermore, at some what lower magnetic fields, the density of states in this subband reaches its maximum just before it is emptied implying a maximum in the intersubband scattering rate as the subband edge crosses the Fermi energy. We can then calculate $E_{n+1}-E_{n}$ from equation 4 and 5 at a magnetic field where a maximum occurs using the measured carrier density. We assign the maximum at the highest magnetic field to the situation where the second subband is emptied. $E_{n+1}-E_{n}$ as obtained from this analysis is shown by the squares in Fig. 4. and the results agree within experimental error with those obtained for $B_{1}$. In addition the calculated magnetic field positions for the coincidence of higher subband edges with the Fermi energy using this value for $E_{n+1}-E_{n}$ agree within measuring accuracy with the resistance maxima observed at lower fields.

In summary it is states that clear quant um oscil. lations occur in a thin $n$-GaAs layer sandwiched between 
p-GaAs layers. The oscillations show a strong anisotropy with respect to the orientation of the magnetic field, and depend on the carrier density which could be varied a factor two with the $U_{n p}$ voltage. These observations are explained by the two-dimensional nature of electronic system in this structure. The separation between the subbands as a function of the carrier density is obtained in a consistent manner from measurements with the magnetic field parallel and perpendicular to the layer plane, under the assumption that the subbands are equidistant. The result (Fig. 4) shows a decreasing subband separation with increasing carrier density as would be expected. Furthermore a subband separation of $35 \mathrm{meV}$ is expected if the depletion layer thickness is exactly half of the doping layer thickness, which corresponds to zero carrier density in the $n$-layer. This value is compatible with an extrapolation of the data in Fig. 4 to $N_{s}=0$. Therefore the results obtained for the subband separation are in good agreement with reasonable assump. tions.

Acknowledgement - We gratefully acknowledge the expert help of I. Jungbauer and B. Kübler for Hall bar and metal contact fabrication. Part of this work was sponsored by the Bundesministerium für forschung und Technologie of the Federal Republic of Germany.

\section{REFERENCES}

1. T. Ando, A.B. Fowler \& F. Stern, Rev. Modern Phys. 54, 437 (1982).

2. G.H. Döhler, H. Künzel, D. Olego, K. Ploog, P. Ruden, H.J. Stolz \& G. Abstreiter, Phys. R?v. Lett. 47, 864 (1982).

3. L. Esaki \& R. Tsu, IBM J. Res. Dev. 14,61 (1970)

4. G.H. Döhler, Phys. Status Solidi (b) 53,73,533 (1972);J. Vac. Sci. Technol. 16,851 (1979).

5. G.H. Döhler, H. Künzel \& K. Ploog. Phys. Rev. B25, 2616 (1982).

6. K. Ploog. H. Künzel, J. Knecht, A. Fischer \& G.H. Döhler, Appl. Phys. Lett. 38, 870 (1981).

7. J.C. Maan, Th. Englert, Ch. Uihlein, H. Künzel, K. Ploog \& A. Fischer, J. Vac. Sci. Technol. (to be published).

8. K. Ploog. A. Fischer \& H. Künzel, J. Electrochem. Soc. $128,400(1981)$.

9. P. Ruden \& G.H. Döhler (to be published).

10. Ch. Zeller, B. Vinter, G. Abstreiter \& K. Ploog, Phys. Rev. B26, 2124 (1982).

11. H.L. Störmer, A.C. Gossard \& W. Wiegman, Solid State Commun. 32, 707 (1982).

12. R.E. Doezema, N. Nealon \& S. Whitmore, Phys. Rev. Lett. 45, 1593 (1980).

13. T. Ando \& Y. Uemura, J. Phys. Soc. Japan 36, 959 (1974).

14. Th. Euglert, D.C. Tsui, A.C. Gossard \& Ch. Uihlein, Surf. Sci. 113, 295 (1982). 